\title{
An Infrared Sensor for Monitoring Meibomian Gland Dysfunction
}

\author{
K. Murawski ${ }^{a, *}$, R. RóżYCKi ${ }^{b}$, P. Murawski ${ }^{c}$, A. Matyja ${ }^{b}$ And M. RęKAs ${ }^{b}$ \\ ${ }^{a}$ Military University of Technology, Institute of Teleinformatics and Automatics \\ S. Kaliskiego 2, 00-908 Warsaw, Poland \\ ${ }^{b}$ Military Institute of Medicine, Ophthalmology Department, Szaserów 128, 04-141 Warsaw, Poland \\ ${ }^{c}$ Military Institute of Medicine, Information and Communication Technology Department \\ Szaserów 128, 04-141 Warsaw, Poland
}

\begin{abstract}
Meibomian gland dysfunction is one of the most common eye disorders observed in clinical practice. It applies to almost $50 \%$ of the population, especially people using contact lenses. It is believed that meibomian gland dysfunction is the most common cause of abnormal stability and integrity of the tear film. Despite this, there is no commercially available equipment for the diagnosis. The article proposes the construction of an optical sensor and a computer system for the rapid, non-invasive diagnosis of meibomian gland dysfunction. The designed hardware and software as well as preliminary results of clinical research are also described.
\end{abstract}

DOI: 10.12693/APhysPolA.124.517

PACS: 42.30.Sy, 07.07.Df, 42.30.Va

\section{Introduction}

Meibom gland dysfunction (MGD) is understood as the diffuse abnormality of the gland. Typical symptoms of MGD are: closing the estuaries of glands or qualitative and quantitative changes in the secretion of meibum. It is believed that MGD affects $39 \%$ to $50 \%$ of individuals of the population. Despite the universality of this disease a relatively small number of major clinical research was done. However, the need to provide detailed research is noticed. Based on literature [1-5] we can say that meibomian glands dysfunction is the most common cause of problems of stability and integrity of the tear film, which adversely affects the performance of the eye. Therefore, it is appropriate to develop methods of diagnosis of meibomian glands. The purpose is to develop devices and diagnostic tests sufficient to clearly identify the degree of gland dysfunction.

The currently used research techniques are mostly focused on the assessment of the edge of the eyelid. Especially when taking into account: the widening of the blood vessels, clogging of the glands, shifting the border between the skin and conjunctiva, observing the changes in the corneal epithelium, tear film break time (BUT), analysis of the mucus of meibomian glands and others (including subjective opinions). More advanced assessment techniques of meibomian glands use: meibography, meibometry [3], interferometry [4] and evaporometry [5]. Meibography is particularly interesting from the mentioned methods.

It allows you to simultaneously assess the number of

*corresponding author; e-mail: k.murawski@ita.wat.edu.pl meibomian glands and their blood supply. The traditional method is an invasive technique. It requires the use of a light source that allows to obtain the phenomenon of transillumination. For this purpose usually the red light is used [6] having a wavelength $\lambda$ from $630 \mathrm{~nm}$ to $770 \mathrm{~nm}$ or the infrared light for which $\lambda$ is in range 780$1400 \mathrm{~nm}$. In view of invasiveness, the central part of the eyelid shall be tested, where the evaluation is regarded as a reference point. In the paper we propose a sensor construction for non-invasive research of the meibomian glands. The sensor design is based on a detector (camera operating in the near infrared) and an IR illuminator precisely controlled by a microcontroller. By taking advantage of the phenomenon that the blood absorbs the heat generated by light in the field of near-infrared, we obtain the imaging of meibomian glands, Fig. 1, which is subject to assessment.

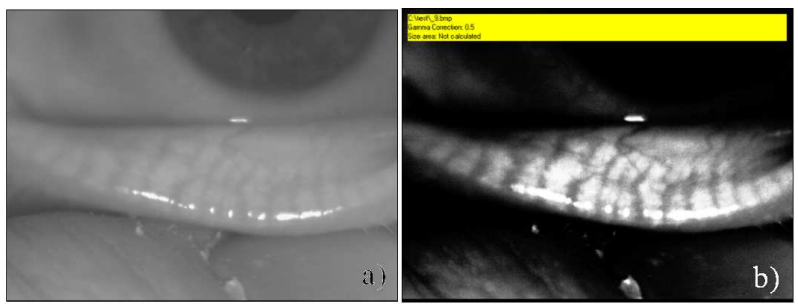

Fig. 1. Images demonstrating a representative view of the Meibom gland: original image (a) and image after processing (b).

To assess the degree of degradation of the meibomian glands we used a three-step scale - meiboscore [4]: degree 1 - loss of less than $1 / 3$ of the area, degree $2-$ the loss from $1 / 3$ to $2 / 3$ of the area, degree 3 - the loss of more than $2 / 3$ of the gland area. 


\section{Optical sensor for monitoring of meibomian gland dysfunction}

The experiment tested two optical systems. The first (prototype), Fig. 2a, was built from a infrared detector (camera Optitrack V120: SLIM) IR pass filter, infrared mirrors and illumination $(\lambda=830 \mathrm{~nm})$. Using the infrared mirror was dictated by structural considerations. Its task was to direct infrared light reflected from the surface of the eye to the detector. The created optical system, along with the emitter and the power supply unit, has been mounted in the housing, Fig. $2 \mathrm{~b}$.
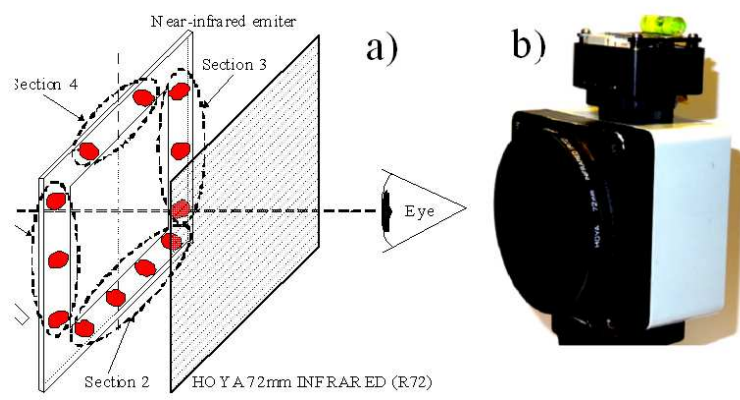

Fig. 2. Preliminary sensor: configuration (a), view (b).

The housing is provided with a bracket for mounting the sensor on a slit lamp. This allows the doctor to use the device in the same way as a typical slit lamp is used. The illuminator is made in the form of a circuit printed in the shape of a frame, Fig. 2a. Twelve TSHG8200 LEDs are placed on it. LEDs are arranged in four groups respectively containing $3,4,3$, and 2 infrared emitters. Sections were included in separate, controlled current sources constructed based on the LM317 system Fig. 3. Each of them were supplied with a constant voltage of $V_{1}=12 \mathrm{~V}$.

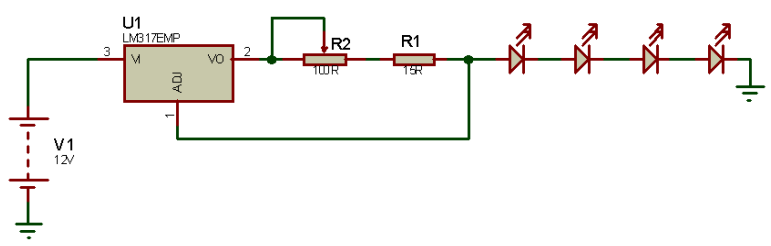

Fig. 3. Control system for one of the IR LED sections.

Resistor values of the IR emitter control system were set so that the minimum forward current $I_{\mathrm{F}}$ of the LEDs equalled $I_{\mathrm{MIN}} \approx 10 \mathrm{~mA}$, and the maximum forward current value $I_{\mathrm{F}}$ did not exceed $I_{\mathrm{MAX}} \approx 85 \mathrm{~mA}$, Fig. 4 .

Even though the designed system was technically correct, in practice it caused many difficulties. The main inconvenience was caused by the need to adjust the illuminator current values at every powering of the device. The continuous changing of the setting values resulted from the way the sensor was stored, which did not guarantee physical separation of the potentiometer knobs from
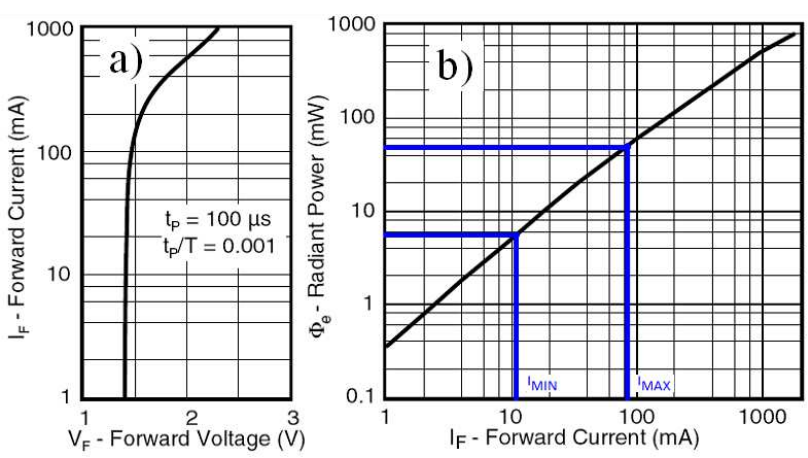

Fig. 4. TSHG8200 diode characteristics: forward current vs. forward voltage (a), radiant power vs. forward current (b).

other elements. This disadvantage was eliminated by designing a microcontroller-controlled version of the device. Other construction adjustments were simultaneously introduced.

The method of mounting the sensor to the slit lamp was modified, this way could give up the infrared mirror. As a result, the cost of the sensor was reduced, and its compact design achieved. Support for the foot switch was added and the ability to save images when pressed was introduced. The radiator system is enriched with two fixation diodes $(\lambda=565 \mathrm{~nm})$, where the patient has to focus his/her eyes on during the test.

The final version of the sensor to observe and study the surface of the eyelids was built based on the Optitrack V120: SLIM camera. V120 was fully configurable via USB interface. It allows acquiring images at a rate of up to $120 \mathrm{fps}$. The sensor design allows dynamic through changing the program of the type of filter blocking IR light passing the IR filter and vice versa. This allows using one sensor to observe the object in infrared and visible light. The camera was closed in a metal housing, which was also the mounting panel for the controller, illuminator, and slit lamp holder, Fig. 6b. The resulting sensor became the basis of the measurement system of MGD.

In the system, Fig. 5a, all the components were combined with a USB interface. As the hub of the system an IBM PC type notebook was used. The result is a diagnosis station for testing the morphology of the Meibom eyelid gland, Fig. 5b.

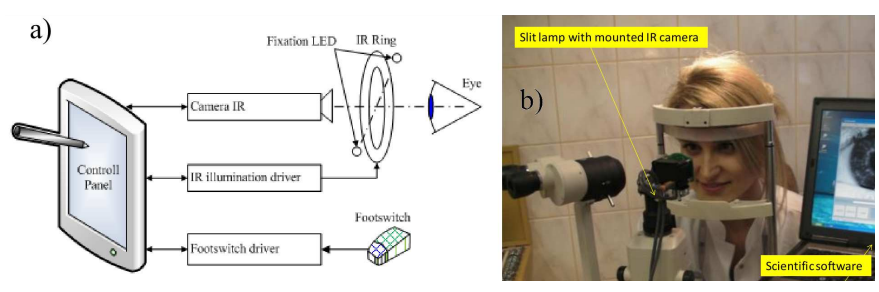

Fig. 5. Measuring system configuration (a), view of the system (b). 
The built system is equipped with specialized software. It automatically initiates the initial values of the infrared detector, the illuminator parameters and it supports the acquisition and analysis of images. It also allows changing the LED IR illuminator brightness and the camera settings. These include: acquisition time, sensitivity, image resolution, fps, etc. The resulting system has been tested in terms of user safety; the results are included in [7].

\section{Software controlling sensor parameters and image acquisition}

The main task of the software controlling the sensor was to make image acquisition and image analysis as well as allowing the changing of illuminator parameters. The ease of use was achieved by creating GUI application software, Fig. 6, operating under a Microsoft Windows class operating system. The application automatically checks for the presence of attached components and initiates them upon start-up. Checked are: the type of infrared detector, IR emitter driver type, and footswitch controller. If all components are correctly identified and initiated the user (doctor) may proceed with the examination.

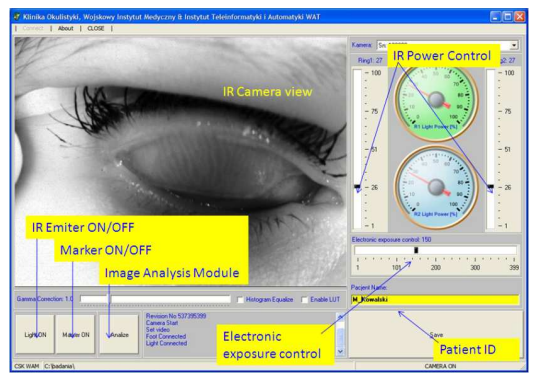

Fig. 6. View of the application for the testing of meibomian gland dysfunction.

The main part of the application was intended to visualize the image of the meibom eyelid glands observed in the infrared band, Fig. 6. This image was processed using techniques [8] that allowed to best highlight them. The standardization of image pixel values, histogram equalization, segmentation, gamma correction, image filtering, and more were implemented in the software. On the right side of the window there are sliders and clocks showing the current power setting of the IR emitter. The power is expressed as a percentage. The function keys that are at the bottom of the screen act as a diagnostics function. They allow checking the operation of the sensor, emitter control system, and the created software. The "analyze" button switches the application view from the image acquisition window to the "image analysis" window, Fig. 6 b. Available features allow instant visualization of the picture meibomian glands, select and automatically calculate: the surface area of the eyelid glands and the area of the gland dysfunction, as well as to express the resulting degree of dysfunction of the glands in a three-step scale - meiboscore.

\section{Object of research, preliminary results}

The aim of the research was to preliminary assess the use of meibography in the diagnosis of chronic eyelid inflammation. The study included a total group of 10 patients (40 eyes) ( 7 men and 3 women) with an average age of $65.7 \pm 13$ with chronic inflammation rims of eyelids and compared the results with a group of 10 healthy volunteers (40 eyes) (4 men and 6 women) in a comparable age group (average age $66.5 \pm 11$ ). Patients were divided into two groups. In all patients the following parameters were examined: the edge of the eyelids and the evaluation of the ocular surface epithelium using a slit lamp, assess the nature of glandular secretions and meibography. After unfolding the eyelid, using the developed sensor, the morphology of the meibomian gland is examined. Partial or complete loss of the meibomian gland was evaluated for each eyelid in a scale: 1 point - loss of less than $1 / 3$ of the total surface of the gland, 2 points - loss of $1 / 3$ to $2 / 3$ and 3 points when the loss covered an area of more than $2 / 3$ of the entire area of the meibomian glands. The result of the meibography that includes the upper and lower eyelids, was prepared on a scale of 0 to 6 points for each eye. Possible stages of the meibomian glands dysfunction, recorded by the developed sensor, are shown in Fig. 7.

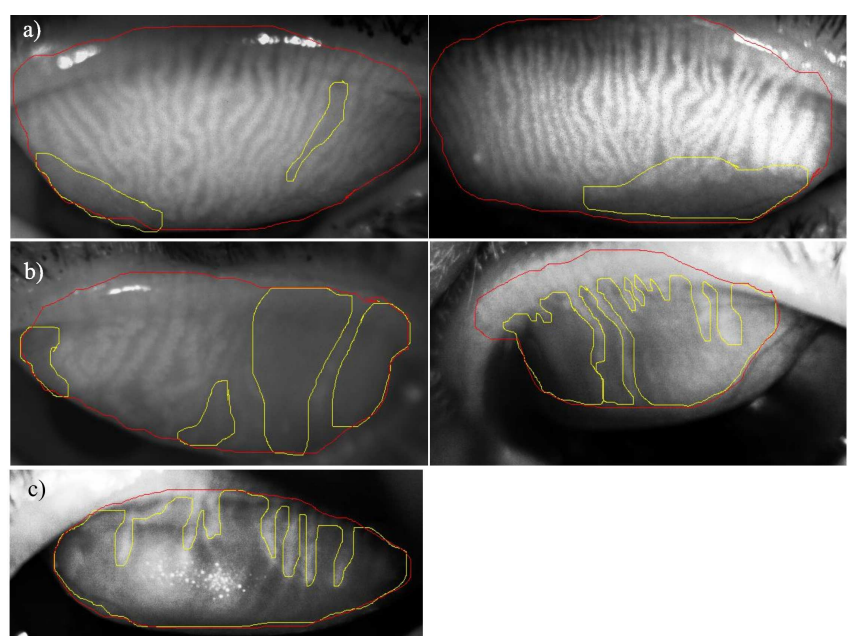

Fig. 7. Exemplary test results: 1 point meiboscore (a), 2 points meiboscore (b), 3 points meiboscore (c).

The results were statistically analyzed. The study was conducted using the Kolmogorov-Smirnov test (Table I) and the Mann-Whitney U (Table II) to achieve statistically significant results at the level of significance of $p<0.050$. Analysis result clearly indicates the correctness of the choice of control and treated groups, Fig. 8a. The high value of correlation is also noticeable between the results obtained using three equal MGD research techniques: study of secretions, Fig. 8b; the study of ocular symptoms, Fig. 8c, and meibography, Fig. 8d. The results show that the constructed sensor provides information non-invasively on the degree of morphological 


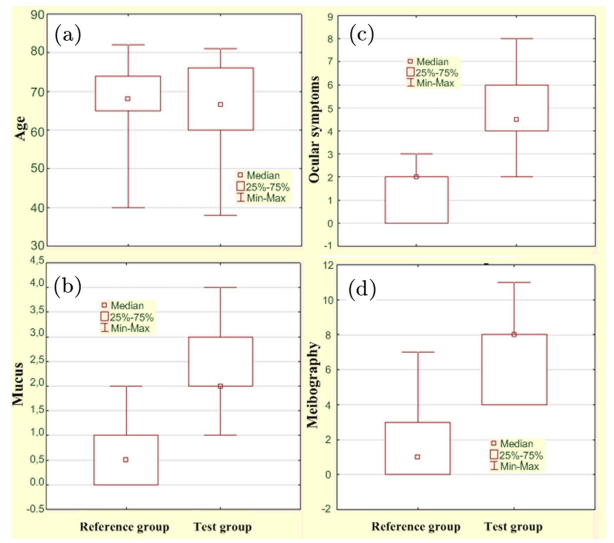

Fig. 8. The results of statistical analysis of obtained data. change of the meibomian glands. It also allows objectively determining the gravity of the disorder. Storing and being able to compare images and numerical results allow the assessment of the effectiveness of medication and undertaken therapy.

\section{Conclusion}

The study shows that the utilization of the developed sensor for meibography research is very promising. The obtained results show that it is possible to use infrared light for testing meibomian glands. Research performed in a significant manner helps in the evaluation of meibomian glandular disorders. Conducted experiments have shown that the chosen sensor configuration is so versatile that it allows you to explore the other features of the eye [9].

Kolmogorov-Smirnov test. Marked in boldface are the results of significance $p<0.050$.

TABLE I

\begin{tabular}{|c|c|c|c|c|c|c|c|c|c|}
\hline \multirow[b]{2}{*}{ Variable } & \multirow[b]{2}{*}{$\begin{array}{l}\text { Max. negative } \\
\text { difference }\end{array}$} & \multirow[b]{2}{*}{$\begin{array}{l}\text { Max. positive } \\
\text { difference }\end{array}$} & \multirow[b]{2}{*}{$p$} & \multicolumn{3}{|c|}{ Control group } & \multicolumn{3}{|c|}{ Test group } \\
\hline & & & & Mean & $\begin{array}{l}\text { Standard } \\
\text { deviation }\end{array}$ & $\begin{array}{c}\text { No. of } \\
\text { valid tests }\end{array}$ & Mean & $\begin{array}{l}\text { Standard } \\
\text { deviation }\end{array}$ & $\begin{array}{c}\text { No. of } \\
\text { valid tests }\end{array}$ \\
\hline Age & -0.20 & 0.20 & $p>0.100$ & 66.50 & 11.95594 & 10 & 65.70 & 13.33375 & 10 \\
\hline No. of symptoms & -0.80 & 0.00 & $p<0.005$ & 1.30 & 1.15950 & 10 & 4.90 & 2.13177 & 10 \\
\hline Examination of secretions & -0.80 & 0.00 & $p<0.005$ & 0.60 & 0.69921 & 10 & 2.30 & 0.82327 & 10 \\
\hline Meibography testing & -0.80 & 0.00 & $p<0.005$ & 1.90 & 2.23358 & 10 & 7.10 & 2.51440 & 10 \\
\hline
\end{tabular}

Mann-Whitney $U$ test. Marked in boldface are the results of significance $p<0.050$.

TABLE II

\begin{tabular}{|c|c|c|c|c|c|c|c|c|c|c|}
\hline \multirow[b]{2}{*}{ Variable } & \multicolumn{2}{|c|}{ Control group } & \multicolumn{2}{|c|}{ Test group } & \multicolumn{6}{|c|}{ Test parameters } \\
\hline & $\begin{array}{c}\text { The sum } \\
\text { of the ranks }\end{array}$ & $\begin{array}{c}\text { No. of } \\
\text { valid tests }\end{array}$ & $\begin{array}{c}\text { The sum } \\
\text { of the ranks }\end{array}$ & $\begin{array}{c}\text { No. of } \\
\text { valid tests }\end{array}$ & $U$ & $Z$ & $p$ & $\begin{array}{c}Z \\
\text { corrected }\end{array}$ & $p$ & $\begin{array}{c}\text { two-tailed } \\
\text { tests }\end{array}$ \\
\hline upper eyelid OP & 67.00 & 10 & 143.00 & 10 & 12.000000 & -2.834730 & 0.004587 & -2.986820 & 0.002819 & 0.002879 \\
\hline upper eyelid OL & 61.00 & 10 & 149.00 & 10 & 6.000000 & -3.288290 & 0.001008 & -3.436150 & 0.000590 & 0.000325 \\
\hline lower eyelid OL & 69.00 & 10 & 141.00 & 10 & 14.000000 & -2.683550 & 0.007285 & -2.825170 & 0.004726 & 0.005196 \\
\hline
\end{tabular}

\section{Acknowledgments}

The Statistical Analysis Software StatSoft, Inc. (2011) STATISTICA (data analysis software system), version 10 was co-financed by the Polish Ministry of Science grant and by the European Union through the European Regional Development Fund under the Innovative Economy Program. Development projects contract no. POIG.02.03.00-00-042/09.

\section{References}

[1] R. Arita, K. Itoh, K. Inoue, S. Amano, Ophthalmology 116, 379 (2009).

[2] R. Arita, K. Itoh, K. Inoue, S. Amano, Ophthalmology 115, 911 (2008).

[3] P. Versura, V. Profazio, E.C. Campos, Acta Ophthalmol. 88, 246 (2010).
[4] escrs.org/publications/eurotimes/13June/ MEIBOMIAN-GLAND .pdf; A. Tomlinson, A.J. Bron, D.R. Korb, S. Amano, J.R. Paugh, E.I. Pearce, R. Yee, N. Yokoi, R. Arita, M. Dogru, Invest. Ophthalmol. Vis. Sci. 52, 2006 (2011).

[5] J.P. McCulley, W.E. Shine, J. Aronowicz, D. Oral, J. Vargas, Trans. Am. Ophthalmol. Soc. 101, 141 (2003).

[6] J. Kasprzak, D. Samsel, A. Borkowska, M. Kecik, Acta Phys. Pol. A 120, 678 (2011).

[7] M. Życzkowski, M. Szustakowski, K. Różanowski, K. Murawski, M. Karol, P. Markowski, Acta Phys. Pol. A 122, 942 (2012).

[8] K. Murawski, K. Różanowski, Acta Phys. Pol. A 124, 509 (2013).

[9] K. Różanowski, K. Murawski, Acta Phys. Pol. A 124, 558 (2013). 